FUNCTIONAL BOWEL DISEASE

\title{
Familial aggregation of irritable bowel syndrome: a prospective study
}

\author{
J S Kalantar, G R Locke III, A R Zinsmeister, C M Beighley, N J Talley
}

See end of article for authors' affiliations Correspondence to: Professor N J Talley, Mayo Clinic, 200 First St SW Rochester, MN 55905, USA; talley.nicholas@ mayo.edu

Accepted for publication 25 June 2003
Background: Patients with irritable bowel syndrome (IBS) often report family members with similar symptoms, but family studies are lacking. We hypothesised that if there is familial aggregation, there would be an increased frequency of IBS in first degree relatives of IBS patients compared with relatives of controls (the patient's spouse).

Methods: A valid self report bowel disease questionnaire (BDQ) that recorded symptoms, the somatic symptom checklist (a measure of somatisation), and a family information form (FIF) to collect the names and addresses of all first degree relatives were mailed to two groups of patients and their spouses (patients attending an IBS educational programme and residents of Olmsted County, Minnesota, who had been coded as IBS on a database). A BDQ was then mailed to all first degree relatives of subjects identified from the FIF. IBS diagnosis in the relatives was based on the Manning criteria.

Results: The BDQ was sent to a total of 355 eligible relatives; $71 \%$ responded $(73 \%$ relatives of patients, $67 \%$ relatives of spouses). Relatives were comparable in mean age, sex distribution, and somatisation score. IBS prevalence was $17 \%$ in patients' relatives versus $7 \%$ in spouses' relatives (odds ratio adjusted for age and sex $2.7(95 \%$ confidence interval $(\mathrm{CI}) 1.2,6.3))$. When also adjusted for somatisation score, the odds ratio was reduced to $2.5(95 \% \mathrm{Cl} 0.9,6.7)$.

Conclusions: Familial aggregation of IBS occurs, supporting a genetic or intrafamilial environment component, but this may be explained in part by familial aggregation of somatisation.
$\mathrm{T}$ he irritable bowel syndrome (IBS) is a symptom complex characterised by abdominal pain or discomfort with disturbed defecation, in the absence of structural or biochemical abnormalities that can be identified utilising currently available tests. ${ }^{12}$ Approximately $7-20 \%$ of the general population experience IBS-like symptoms. ${ }^{3}$ The cost of IBS is high in terms of health care utilisation (outpatient costs, hospitalisation costs, prescription costs) and employer costs. ${ }^{5}{ }^{6}$ IBS accounts for $25-50 \%$ of referrals to gastroenterologists, 96000 hospital discharges, three million physician visits, and 2.2 million prescriptions annually in the USA. ${ }^{6}$ Numerous studies have shown that the quality of life of individuals with IBS is lower than the general population, and even lower than individuals with congestive heart failure. ${ }^{7}$ Many patients with IBS have multiple non-gastrointestinal symptoms (for example, fatigue, musculoskeletal pain), and while this association is unexplained it can confound epidemiological association studies. ${ }^{2}$

Epidemiological and clinical studies have identified a number of functional abnormalities and other features associated with functional gastrointestinal disorders. These include abnormalities of gastrointestinal motor or visceral sensory function, ${ }^{28}$ minimal inflammation post-infection, ${ }^{9}$ and psychological disturbances such as depression or anxiety disorders. ${ }^{10}$ These disorders are frequently considered to be the result of disturbed brain-gut interactions; $;^{12}$ brain imaging studies of cerebral blood flow have consistently suggested that these are central disturbances of afferent signal processing in IBS, although whether there findings are unique in IBS remains to be clarified. ${ }^{211-13}$ Much emphasis has also been placed on disturbances of peripheral motor and sensory function in IBS but whether colonic pain sensitivity to distension is a biological marker is in dispute. ${ }^{214}$ Intrafamilial learning and genetics could both play an important role in the pathogenesis of IBS. Several of our studies have provided evidence for a genetic contribution to IBS, ${ }^{15-17}$ although whether genetic factors modulate the documented sensorimotor or central disturbances in IBS is currently unknown. Indeed, a genetic association is controversial, as not all twin studies have confirmed the observations. ${ }^{18}$ There is also evidence of a strong environmental contribution to IBS, with social learning early in life and infection being implicated. ${ }^{2}{ }^{17}$

In a cross sectional study, we observed that patients who reported a family member with abdominal pain or bowel problems were at a twofold increased risk of reporting symptoms of IBS. ${ }^{15}$ This may be explained by genetic or common environmental factors, or both. However, the validity of the subjects' reporting of family members' symptoms is not known, and other family studies are lacking.

If familial aggregation exists, this would provide the rationale to search for both genetic and intrafamilial environmental causes. Whether familial aggregation of IBS occurs is, nevertheless, uncertain. We hypothesised that there is an increased frequency of IBS in first degree relatives of IBS patients compared with relatives of controls (the patient's spouse), because IBS is, in part, inherited.

\section{METHODS}

\section{Probands}

Two groups of patients with IBS were identified: (1) patients attending the Mayo Clinic IBS educational programme and (2) residents of Olmsted County, Minnesota, USA, who had been recently coded with an IBS diagnosis. The IBS educational programme is a structured three hour one time

Abbreviations: IBS, irritable bowel syndrome; $B D Q$, bowel disease questionnaire; FIF, family information form 
class with 1-6 IBS patients attending daily who are referred by a Mayo physician after evaluation. ${ }^{19}$ The Olmsted County IBS patients were identified using the diagnostic index of the Mayo Clinic. The Olmsted County population comprises approximately 100000 persons of whom 89\% are White; sociodemographically, the community is similar to the USA White population. ${ }^{20}$ Eighty per cent of the Olmsted County population resides within five miles of Rochester, and residents receive their medical care almost exclusively from two group practices: the Mayo Medical Center and Olmsted Medical Center. The Mayo Clinic has maintained a common medical record system with its two affiliated hospitals (Saint Mary's and Rochester Methodist) for over 90 years. Recorded diagnoses and surgical procedures are indexed, including diagnoses made for outpatients seen in office or clinic consultations, emergency room visits or nursing home care, as well as diagnoses recorded for hospital inpatients, at autopsy examination, or on death certificates..$^{20}$ Thus details of the medical care provided at the Mayo Clinic to residents of the county are available for study. The IBS class sample was drawn in random order from a data set of subjects from a previous consecutive sample of class attendees. The medical records sample was drawn in random order from all Olmsted County residents given an IBS diagnosis at the Mayo Clinic over a two year period. Patients were mailed recruitment information until 35 in each group had responded and were eligible.

To be eligible for this study, the patient had to be married and aged 18-80 years. Patients with a psychiatric disease, nursing home residents, and people living outside the USA were excluded.

\section{Questionnaires}

A valid self report bowel disease questionnaire (BDQ) that recorded gastrointestinal symptoms and the somatic symptom checklist (a measure of somatisation) was used. ${ }^{21}$ The somatic symptom checklist measures the frequency and bothersomeness of 12 non-gastrointestinal symptoms on a five level graded Likert scale. The overall mean score is computed for each subject; we used this to adjust for the tendency to be a high symptom reporter (somatiser). In addition, a family information form (FIF) to collect the names and addresses of all first degree relatives was used. The FIF was developed and used in a previous study of familial aggregation of reflux..$^{22}$ The form specifically asks for biological (blood) relatives and instructs the respondent not to include half siblings. The names of all such relatives were requested regardless of vital status. A separate question asked the vital status (alive, dead) of each relative.

\section{Survey of patients and spouses}

Two copies of the BDQ and FIF were mailed to IBS patients. A cover letter allowed the opportunity to refuse participation. The instructions asked the patient and their spouse to each independently complete a BDQ and FIF, and mail them back in a postage paid self addressed envelope that was provided. Reminder letters and new copies of the BDQ and FIF were sent to non-responders after three and six weeks.

\section{Information from relatives}

The BDQ was mailed to all living first degree relatives (siblings, parents, children) of the patient and spouse, as identified on the FIF. A cover letter asked for their participation and allowed the opportunity to refuse participation and no longer be contacted. A postage paid return envelope was provided. Reminder letters with copies of the BDQ and FIF were sent after three weeks and six weeks to all non-responders.

\section{Diagnostic criteria}

Symptom reporting on the BDQ was used to make a diagnosis of IBS. IBS was defined based on the Manning criteria $^{15}{ }^{16}$ or the Rome I criteria for IBS. ${ }^{1}$ The Manning criteria required abdominal pain plus at least two of the following six symptoms: pain relief by defecation; looser stools at the onset of pain; more frequent stools at the onset of pain; abdominal distension; mucus per rectum; and feelings of incomplete evacuation. ${ }^{23}$ We have used these criteria in previous epidemiological studies and have shown that the approach is robust compared with the Rome criteria. $^{24-27}$ The Rome I criteria used here comprised abdominal pain relieved with defecation and/or associated with a change in stool frequency $(>3$ per day or $<3$ per week) and/or associated with a change in stool consistency (loose/watery or hard, often), plus two or more of: altered stool frequency, altered consistency, altered stool passage (straining, urgency, or feelings of incomplete evacuation), mucus, or bloating with distension, often. ${ }^{1}$ The BDQ measures each of these symptom components and the criteria were combined using a standard algorithm. ${ }^{27}$

\section{Statistical analyses}

Demographics for patients, spouses, and their relatives were summarised by calculating means (SD) (or median (range)) for continuous variables (for example, age and somatisation score) and proportions for categorical variables (for example, sex, IBS). The distribution of age was symmetric while that for the somatisation score was somewhat positively skewed. All subjects that met the Rome I criteria for IBS also met the Manning criteria, thus all analyses were based on the Manning criteria. Spouses who met IBS criteria were excluded from the analyses, along with their family members. The IBS status in relatives of families that had both a patient and qualifying spouse (that is, for "matched" sets of relatives) was assessed using matched logistic regression models taking into account the correlation within families via the generalised estimating equations approach. This analysis aimed to assess the odds for IBS in the patients' family members relative to spouse family members (that is, to assess whether there was an association between individual subject IBS status and being a member of a patient family versus a spouse family member). Two additional models adjusting for age and sex, and separately, age, sex, and somatisation score were also examined. Additional analyses ignoring the matching were done using data from all patient and qualified spouse families applying similar logistic regression methods. As an alternate approach, a separate matched logistic regression analysis was examined using families rather than individuals as the unit of analysis. This approach was examined to avoid the need to choose a correlation structure among family members. This analysis assessed the association of (family) status (that is, patient family as opposed to spouse family) versus whether the family had at least one member with IBS. The total number of family members providing response data was also included as a covariate in these analyses.

The anticipated prevalence of IBS in spouses' relatives was $15 \%$, and the anticipated prevalence of IBS in patients' relatives was $30 \%$. Based on these anticipated prevalence rates, it was estimated that a sample size of 100 relatives for both the patient and spouse would provide statistical power of $80 \%$ (alpha $=0.05$ ) to detect an association corresponding to a difference of $15 \%$ versus $32 \%$ in the proportion of subjects with IBS based on a two sample test for proportions. Previous experience suggested that each patient and spouse would have approximately three relatives, thus 35 subjects in each case and control group were estimated to be needed. Surveys were mailed until this sample size was reached. 


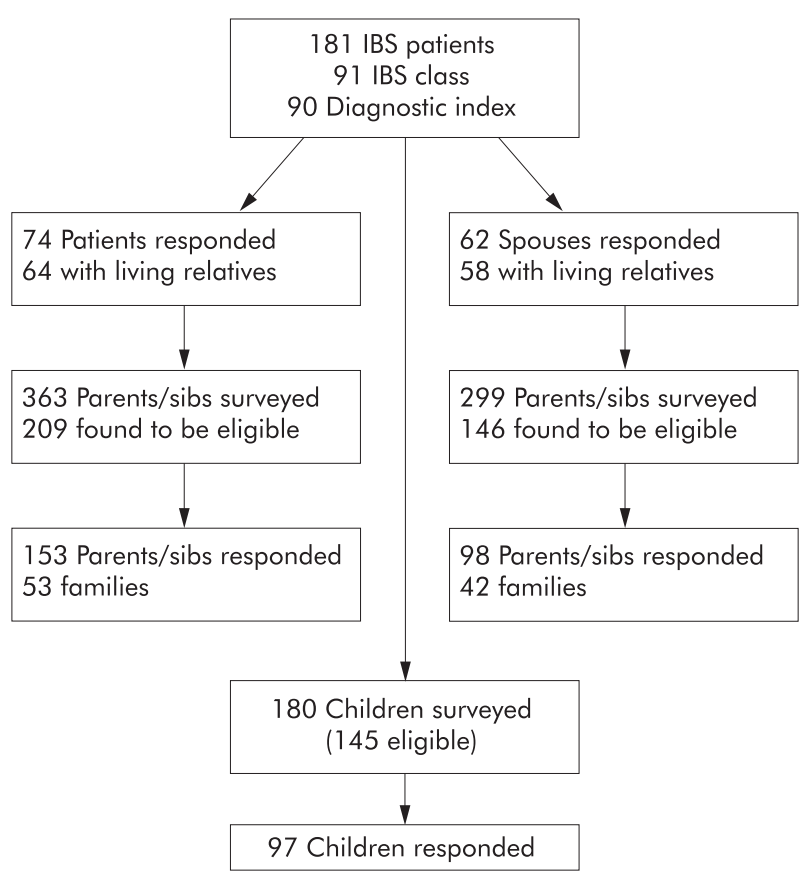

Figure 1 Patient recruitment flow chart. IBS, irritable bowel syndrome.

\section{RESULTS}

\section{Survey response}

The BDQ and FIF questionnaires were sent to a total of 181 patients (91 from the IBS class, 90 from Olmsted County). Recruitment of subjects is illustrated in fig 1. Of these 181, a total of $74(41 \%)$ responded of whom $64(86 \%)$ had living relatives. Of the 74 patients, $62(84 \%)$ had spouses who responded and 58 (94\%) of these had living relatives. Surveys were then sent to 363 parents or siblings of patients, 299 parents or siblings of spouses, and 180 children of the patients and a spouse. Responses were received from 153 of the patients' relatives, 98 of the spouses' relatives, and 97 of the children. An overall response rate of $71 \%$ was achieved (73\% in relatives of patients, $67 \%$ in relatives of spouses). Patients, spouses, and their relatives were similar in mean age, sex distribution, and somatisation score (table 1). Of patients with a clinician based IBS diagnosis, $62 \%$ met the Manning or Rome I criteria for IBS, and among the spouses, $8 \%$ met the Manning or Rome I criteria for IBS. The families for these five spouses that met criteria for IBS were removed from all subsequent analyses.

\section{IBS in patients and families}

In the matched analyses where data were available from both families of the patient and spouse, the prevalence of IBS was
$17 \%$ in patients' relatives versus $7 \%$ in spouses' relatives (odds ratio 2.72 (95\% confidence interval (CI) 1.19, 6.25)) (table 2). Thus there was a significant increase in the prevalence of IBS in patient family members versus spouse family members. This was not changed by adjustment for age and sex; however, after adjusting for somatisation score the odds radio was reduced and was no longer significant (odds ratio 2.51 (95\% CI 0.94, 6.68)). When comparing families (30 families had data from relatives of both the patient and spouse), $40 \%$ of patients' families had at least one member with IBS compared with $17 \%$ in spouses' families (odds ratio 3.36 (95\% CI 0.70, 16.02)). Thus patient families were more likely to have at least one family member with IBS although this was not statistically significant. In the unmatched analyses, using data from all families of patients and qualified spouses, an odds ratio of 2.11 was detected $(95 \%$ CI $0.97,4.55)$.

\section{DISCUSSION}

As far as we are aware, this is the first study to directly evaluate relatives of IBS probands and provide data on familial aggregation. In a previous cross sectional study, patients who reported a family member with abdominal pain or bowel problems were at increased risk (odds ratio 2.3) of reporting symptoms of IBS, ${ }^{15}$ but the validity of the subjects' reporting of family members' symptoms was not known. Here we found that the risk estimate of IBS was similar to our previous self report study (odds ratio 2.7) and this was statistically significant. Similar results were observed when only patient and spouse families were compared although the numbers (and hence the power) were then reduced and the results were not statistically significant.

We applied the Manning criteria rather than the Rome criteria to ensure maximum sensitivity in this study ${ }^{24-27}$ but the prevalence of IBS seen in the families of our patients and spouses was lower than expected. The prevalence of IBS in the community has been assessed in several studies and depends greatly on the diagnostic criteria used. ${ }^{3}$ In our previous work, using a threshold of two of six Manning criteria to make a diagnosis of IBS would lead to a prevalence estimate of 17 per $100 .^{27}$ We estimated the risk would be twofold ${ }^{15}$ and that risk was confirmed. However, rather than finding prevalence rates in families of patients to be higher than the general population, we found the prevalence rates in spouses' families to be lower than the population. One possibility is that relatives with IBS may be less likely to respond; however, the response rates among relatives was adequate $(71 \%)$. Alternatively, it is conceivable that some of the issues related to familial aggregation of IBS may make it less likely in people who are married. Unmarried patients with IBS were not eligible and therefore young people and divorced people were not represented. Notably, IBS is associated with abuse and abuse is associated with

Table 1 Summary of demographic and symptom data in patients, spouses, and relatives

\begin{tabular}{|c|c|c|c|c|c|c|c|c|c|}
\hline & Patients & Spouses & $\begin{array}{l}\text { Patients' } \\
\text { parents/sibs }\end{array}$ & $\begin{array}{l}\text { Patients' } \\
\text { parents }\end{array}$ & $\begin{array}{l}\text { Patients' } \\
\text { sibs }\end{array}$ & $\begin{array}{l}\text { Spouses' }^{\prime} \\
\text { parents/sibs }\end{array}$ & $\begin{array}{l}\text { Spouses' } \\
\text { parents }\end{array}$ & $\begin{array}{l}\text { Spouses' } \\
\text { sibs }\end{array}$ & Children \\
\hline $\mathrm{n}$ & 74 & 62 & 153 & 33 & 120 & 98 & 24 & 74 & 97 \\
\hline$\%$ Female & $57 \%$ & $45 \%$ & $54 \%$ & $61 \%$ & $52 \%$ & $55 \%$ & $67 \%$ & $51 \%$ & $51 \%$ \\
\hline Age $(y)^{*}$ & $58(13)$ & $59(13)$ & $60(15)$ & $75(11)$ & 57 (13) & $62(14)$ & $74(11)$ & $58(13)$ & $38(9)$ \\
\hline Somatisation score $†$ & $\begin{array}{l}1.0 \\
(0.0-2.33)\end{array}$ & $\begin{array}{l}0.54 \\
(0.0-1.88)\end{array}$ & $\begin{array}{l}0.58 \\
(0.0-2.75)\end{array}$ & $\begin{array}{l}0.50 \\
(0.0-2.71)\end{array}$ & $\begin{array}{l}0.58 \\
(0.0-2.75)\end{array}$ & $\begin{array}{l}0.50 \\
(0.0-2.67)\end{array}$ & $\begin{array}{l}0.58 \\
(0.0-1.79)\end{array}$ & $\begin{array}{l}0.48 \\
(0.0-2.67)\end{array}$ & $\begin{array}{l}0.54 \\
(0.04-2.67)\end{array}$ \\
\hline \% with IBS (Rome I) & $45 \%$ & $5 \%$ & $7 \%$ & $3 \%$ & $8 \%$ & $4 \%$ & $0 \%$ & $5 \%$ & $12 \%$ \\
\hline$\%$ with IBS (Manning) & $62 \%$ & $8 \%$ & $13 \%$ & $12 \%$ & $13 \%$ & $7 \%$ & $0 \%$ & $9 \%$ & $20 \%$ \\
\hline
\end{tabular}

*Values are mean (SD).

†Values are median (range).

IBS, irritable bowel syndrome. 
Table 2 Matched family comparisons

\begin{tabular}{|c|c|c|c|c|}
\hline $\begin{array}{l}\text { Unit measured } \\
\text { (n) }\end{array}$ & $\begin{array}{l}\text { \% with IBS } \\
\text { (Manning or Rome I) }\end{array}$ & OR $(95 \% \mathrm{CI})^{*}$ & $\begin{array}{l}\text { Adjusted OR } \\
(95 \% \mathrm{Cl}) \dagger\end{array}$ & $\begin{array}{l}\text { Adjusted OR } \\
(95 \% \mathrm{Cl}) \ddagger\end{array}$ \\
\hline \multicolumn{5}{|l|}{ Individuals } \\
\hline $\begin{array}{l}\text { Patients }(90) \\
\text { Spouses (74) }\end{array}$ & $\begin{array}{r}17 \\
7\end{array}$ & $2.72(1.19,6.25)$ & $2.72(1.18,6.26)$ & $2.51(0.94,6.68)$ \\
\hline \multicolumn{5}{|l|}{ Family } \\
\hline Patients (30) & 40 & $3.36(0.70,16.02)$ & & \\
\hline Spouses (30) & 17 & & & \\
\hline \multicolumn{5}{|c|}{$\begin{array}{l}\text { *When comparing families, a hazard ratio is presented for the number of families with at least one member with } \\
\text { irritable bowel syndrome (IBS), adjusted for the total number of family members. } \\
\text { †Adjusted for sex and age. } \\
\text { †Adjusted for sex, age, and somatic symptom checklist (somatisation) score. }\end{array}$} \\
\hline
\end{tabular}

divorce, $^{28}{ }^{29}$ making this bias tenable. However, we did not restrict selection of relatives by marital status.

The response rate in the initial recruitment of index patients was not as high as was hoped when planning this study. The recruitment rate in this study was lower than the rate obtained in a similar study of gastro-oesophageal reflux. ${ }^{22}$ It is possible that patients with IBS or those from dysfunctional families are less likely to participate by providing information about their family members.

In order to reduce bias, we selected probands with IBS from two sources. Those attending the IBS class reflect a referral population and those from the diagnostic index reflect the local population. The study subjects were the relatives. Their recruitment depended on the proband and spouse being willing to participate and in turn willing to complete a questionnaire. The relatives of the proband and spouse were similar in their response rate and age, sex, and somatisation ratings. It is possible that relatives of patients with gastrointestinal symptoms may be more likely to endorse gastrointestinal symptoms on a questionnaire than relatives of people without gastrointestinal symptoms, or affected relatives of a proband may be more likely to participate in general. However, the low prevalence of IBS symptom reporting in this study suggests these issues were not present.

IBS is more common in women. ${ }^{24-28}$ As expected, the majority of probands were female and the majority of spouses were male. Notably, the sex distribution in siblings was similar in the relatives of probands ( $52 \%$ female) and spouses ( $51 \%$ female), reducing the chance that the increased odds observed were due to sex differences. Age of onset of IBS is not known, although it is thought to be a disease of young adults. ${ }^{2}$ The majority of the study population was over the age of 50 years and thus we suspect that they would have developed IBS by this age. For these reasons, all of our analyses were adjusted for age and sex.

Our results support the hypothesis that there is either a genetic or intrafamilial component in IBS. Several other studies suggest that there could be a genetic component in IBS. Two twin studies have implicated a genetic component; monozygotic twins showed higher concordance than dizygotic twins for IBS. ${ }^{16}{ }^{17}$ In a study in Australia, 686 same sex twin pairs enrolled in the Australian Twin Registry were interviewed and symptoms consistent with functional bowel disorders were derived. ${ }^{16} \mathrm{~A}$ model that most closely fitted the data was obtained in which $57 \%$ of the variance was attributed to additive genetic variance, with the remaining $43 \%$ attributed to the individual's unique environment. A USA study surveying 6060 twin pairs showed 17\% concordance in monozygotic twins and only $8 \%$ concordance in dizygotic twins, further supporting a genetic contribution for IBS. ${ }^{17}$

It is unlikely that a single disease gene could be responsible for IBS-a classic monogenic Mendelian mechanism. Much more likely, multiple genes may collectively cause IBS-a polygenic model of a complex disorder. With either mechanism, gene-environment interactions would be likely to affect phenotypic expression. This may in part explain the variation in symptoms of IBS. A serotonin transporter polymorphism (5HTT-LPR) has been linked to IBS response to therapy. ${ }^{30}$ Camilleri et al showed that serotonin transporter homozygotes for the wild-type (long/long) alleles had slower colonic transit times and were more likely to respond to alosetron, a $5-\mathrm{HT}_{3}$ receptor antagonist. ${ }^{30}$ However, no difference in genotype frequency was seen between the constipation predominant IBS group and healthy controls, and specifically, the long/long genotype frequency was low in the constipation IBS group. The search for multiple polymorphisms that may account for IBS is underway ${ }^{31}$ but searching is likely to be fruitless without candidate gene hypotheses; family studies may in particular provide a resource in terms of enriching the yield of future genetic studies, based on our results that familial aggregation exists. Notably, the twin studies suggest there is also a strong environmental contribution. ${ }^{16}{ }^{17}$ Levy et al have studied children of parents with IBS and controls, and found that cases visited doctors significantly more often, suggesting specific types of illness behaviour may be learned. ${ }^{32}$ In a twins study, Levy et al also observed that having a mother or father with IBS were independent predictors of IBS status, supporting the hypothesis that what an individual learns from their environments has at least an equal influence as heredity in IBS. ${ }^{17}$

Familial aggregation of IBS has been directly demonstrated for the first time but a larger sample size will be necessary to confirm these observations applying the Rome criteria. Furthermore, the current study was not powered to look at differential associations by relative type. The prevalence of IBS was lower than expected; indeed, only $45 \%$ of patients with an IBS diagnosis in the clinic reported IBS symptoms consistent with Rome I criteria. Other studies suggest that the Rome I and II criteria under diagnose IBS; ${ }^{25-27}$ the lack of a diagnostic marker inhibits more accurate identification of the phenotypes in IBS which in turn limits genetic epidemiology studies of this condition. Up to half of all patients with IBS report non-gastrointestinal symptoms consistent with fibromyalgia, chronic fatigue syndrome, chronic pelvic pain, and temporomandibular joint disorder. ${ }^{33}$ It is notable that when we adjusted for somatisation scores (as measured by the somatic symptom index), the association with IBS was 2.5-fold increased but was no longer significant. Hence while IBS aggregates in families, it is conceivable that such aggregation may be explained by familial aggregation of somatisation. Data are limited but Kendler reported that genetic effects were significant for self report symptoms of somatisation in a large twin family sample. ${ }^{34}$ Alternatively, IBS may in fact be part of a systemic symptom disorder that aggregates in families, perhaps related 
to diffuse central neurological dysfunction inducing lower visceral and in some somatic pain thresholds. ${ }^{23}$

In conclusion, this study provides the first data on familial aggregation in IBS based on a direct survey of relatives, and provides key information for planning future research. If familial aggregation is confirmed in future studies, the roles of common intrafamilial environmental as well as inherited factors will both require careful evaluation.

\section{ACKNOWLEDGEMENT}

This study was sponsored in part by Novartis.

\section{Authors' affiliations}

J S Kalantar, Department of Medicine, University of Sydney, Australia G R Locke III, Division of Gastroenterology and Internal Medicine, Mayo Clinic and Mayo Foundation, Rochester, MN, USA

A R Zinsmeister, C M Beighley, Division of Biostatistics, Mayo Clinic and Mayo Foundation, Rochester, MN, USA

N J Talley, Division of Gastroenterology and Internal Medicine, Mayo Clinic and Mayo Foundation, Rochester, MN, USA, and Department of Medicine, University of Sydney, Australia

\section{REFERENCES}

1 Thompson WG, Longstreth GF, Drossman DA, et al. Functional bowel disorders and functional abdominal pain. Gut 1999:45(suppl 2):1143-7.

2 Talley NJ, Spiller R. Irritable bowel syndrome: a little understood organic bowel disease? Lancet 2002;360:555-64.

3 Saito YA, Schoenfeld P, Locke GR III. The epidemiology of irritable bowe syndrome in North America: a systematic review. Am J Gastroenterol 2002;97:1910-15.

4 Barbezat G, Poulton R, Milne B, et al. Prevalence and correlates of irritable bowel symptoms in a New Zealand birth cohort. N Z Med J 2002;1 15:U220.

5 Talley NJ, Gabriel SE, Harmsen WS, et al. Medical costs in community subjects with irritable bowel syndrome. Gastroenterology 1995: 109:1736-41

6 Sandler RS, Everhart JE, Donowitz M, et al. The burden of selected digestive diseases in the United States. Gastroenterology 2002;122:1500-11

7 El-Serag HB, Olden K, Bjorkman D. Health-related quality of life among persons with irritable bowel syndrome: a systematic review. Aliment Pharmacol Ther 2002:16:1171-85.

8 Munakata J, Naliboff $B$, Harraf F, et al. Repetitive sigmoid stimulation induces rectal hyperalgesia in patients with irritable bowel syndrome. Gastroenterology 1997:112:55-63.

9 Neal KR, Barker L, Spiller RC. Prognosis in post-infective irritable bowel syndrome: a six year follow up study. Gut 2002;51:410-13.

10 Drossman DA, McKee DC, Sandler RS, et al. Psychological factors in the irritable bowel syndrome. A multivariate study of patients and nonpatients with irritable bowel syndrome. Gastroenterology 1988;95:701-8.

11 Verne GN, Himes NC, Robinson ME, et al. Central representation of visceral and cutaneous hypersensitivity in the irritable bowel syndrome. Pain 2003; 103:99-110

12 Berman SM, Chang L, Suyenobu B, et al. Condition-specific deactivation of brain regions by $5-\mathrm{HT} 3$ receptor antagonist alosetron. Gastroenterology 2002; 123:969-77.
13 Bonaz B, Baciu M, Papillon E, et al. Central processing of rectal pain in patients with irritable bowel syndrome: fMRI study. Am J Gastroenterol 2002;97:654-61

14 Whitehead WE, Palsson OS. Is rectal pain sensitivity a biological marker for irritable bowel syndrome: psychological influences on pain perception. Gastroenterology 1998;115:1263-71.

15 Locke GR III, Zinsmeister AR, Talley NJ, et al. Familial association in adults with functional gastrointestinal disorders. Mayo Clin Proc 2000;75:907-12.

16 Morris-Yates A, Talley NJ, Boyce PM, et al. Evidence of a genetic contribution to functional bowel disorder. Am J Gastroenterol 1998;93:1311-17.

17 Levy RL, Jones KR, Whitehead WE, et al. Irritable bowel syndrome in twins: heredity and social learning both contribute to etiology. Gastroenterology $2001 ; 121: 799-804$.

18 Mohammed J, Cherkas L, Riley SA, et al. Genetic influence in irritable bowel syndrome: a twin study. Gut 2002;50:A1.

19 Colwell L, Prather CM, Phillips SF, et al. Effects of an irritable bowel syndrome educational class on health-promoting behaviors and symptoms. Am J Gastroenterol 1998;93:901-5.

20 Melton L III. History of the Rochester Epidemiology Project. Mayo Clin Proc 1996;71:266-74.

21 Talley NJ, Phillips SF, Melton LI, et al. A patient questionnaire to identify bowel disease. Ann Intern Med 1989;1 11:671-4.

22 Romero Y, Cameron AJ, Locke GR III, et al. Familial aggregation of gastroesophageal reflux in patients with Barrett's esophagus and esophageal adenocarcinoma. Gastroenterology 1997;10:1449-56.

23 Manning AP, Thompson WG, Heaton KW, et al. Towards positive diagnosis of the irritable bowel. Br Med J 1978;2:653-4.

24 Talley NJ, Zinsmeister AR, Van Dyke C, et al. Epidemiology of colonic symptoms and the irritable bowel syndrome. Gastroenterology 1991;101:927-34.

25 Mearin F, Badia X, Balboa A, et al. Irritable bowel syndrome prevalence varies enormously depending on the employed diagnostic criteria: comparison of Rome II versus previous criteria in a general population. Scand J Gastroenterol 2001;36:1155-61.

26 Boyce PM, Koloski NA, Talley NJ. Irritable bowel syndrome according to varying diagnostic criteria: are the new Rome II criteria unnecessarily restrictive in research and practice? Am J Gastroenterol 2000;95:3176-83.

27 Saito YA, Locke GR, Talley NJ, et al. A comparison of the Rome and Manning criteria for case identification in epidemiological investigations of irritable bowel syndrome. Am J Gastroenterol 2000;95:2816-24.

28 Talley NJ, Fett SL, Zinsmeister AR, et al. Gastrointestinal tract symptoms and self-reported abuse: a population-based study. Gastroenterology 1994:107:1040-9.

29 Drossman DA, Talley NJ, Leserman J, et al. Sexual and physical abuse and gastrointestinal illness. Review and recommendations. Ann Intern Med 1995; 123:782-94.

30 Camilleri M, Atanasova E, Carlson PJ, et al. Serotonin-transporter polymorphism pharmacogenetics in diarrhea-predominant irritable bowel syndrome. Gastroenterology 2002;123:425-32.

31 Pata C, Erdal ME, Derici E, et al. Serotonin transporter gene polymorphism in irritable bowel syndrome. Am J Gastroenterol 2002;97:1780-4.

32 Levy RL, Whitehead WE, Von Korff MR, et al. Intergenerational transmission of gastrointestinal illness behavior. Am J Gastroenterol 2000;95:451-6.

33 Whitehead WE, Palsson O, Jones KR. Systematic review of the comorbidity of irritable bowel syndrome with other disorders: what are the causes and implications? Gastroenterology 2002;122:1140-56.

34 Kendler KS, Walters EE, Truett KR, et al. A twin-family study of self-report symptoms of panic-phobia and somatization. Behav Genet $1995 \cdot 25 \cdot 499-515$. 